\section{The Sterilisation of Liquids by Light of very short} Wave-length.

DURING the past year several articles have appeared in the Comptes rendus des Séances de l'Académie des Sciences, Paris, on the sterilisation of liquids by ultra-violet light. The notes of $\mathbf{M}$. Billon-Daguerre have particularly attracted my attention, since he has endeavoured to utilise the region of the spectrum discovered by Schumann for the sterilisation of water. It is obvious that the question of the transparency of water for light of very short wave-length is important in this connection, and, as there seems to be no data which bears on the matter, I have recently made some experiments.

I used a vacuum grating spectroscope arranged in the same way as when I investigated the transparency of some solid substances. The water was distilled, but without any special precautions, and was enclosed in a cell with fluorite windows. Two of these cells were employed, one giving a water column of half a millimetre, the other giving a millimetre column. With the half-millimetre cell in the light path the spectrum was cut off at $\lambda{ }_{1792}$ (Ångström units), even after a prolonged exposure. It appeared that this limit of the spectrum receded rather slowly toward the red with increase in the thickness of the water column.

As M. Billon-Daguerre wished to use light of very short wave-length, he employed a vacuum tube filled with hydrogen. This substance is known to give a strong spectrum in the region between $\lambda 1650$ and $\lambda$ 1030; it must not be forgotten, however, that no lines can be ascribed to it in the region between $\lambda 2000$ and $\lambda 1650$. Thus any action due to the radiation from the vacuum tube filled with hydrogen must be confined to a layer of water so thin that light of wave-lengths shorter than $\lambda 165^{\circ}$ can penetrate it. Judging from my experiments, such a layer must be very thin indeed.

Several investigators have used the mercury arc in quartz as a source of light in sterilisation experiments. There are two facts which it may be interesting to mention in this connection. In the first place, fused quartz two millimetres thick is somewhat transparent so far as $\lambda$ I 500 ; the transparency falls off rapidly with increasing thickness. In the second place, no lines more refrangible than the strong line at $\lambda_{1} 850$ are known in the spectrum of mercury. In this second statement my own observations are confirmed by a recent investigation of Dr. Handke.

THEODORE LyMAN.

\section{Jefferson Laboratory, Harvard University, July 8 .}

\section{Elemental Weight Accurately a Function of the \\ Volution of Best Space-svmmetry Ratios.}

IT is a fact little known, but of the first magnitude, that equal spheres or corpuscles cannot in space, as in one plane, be distributed at equal mutual distances. Tetrahedra, the four points of which alone are all mutually equidistant, cannot be packed so as to fill space, as their face-angles to fill one plane. ${ }^{1}$ Icosahedral diffusion, with a central sphere, nearly achieves this, but by a cramping of the central point in the ratio $\mathrm{I}: \mathrm{I} \cdot 05 \mathrm{I} 460 \cdots$

Free magnetic needles in water, say five in number, may fall into position either thus :

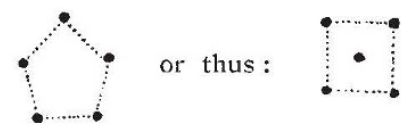

Their energies are a fixed quantity; so that, though they will assume either position, they are stabler in position (a), because here, on the whole, the lines are more equidistant; but $(b)$ might become equally stable if each needle were a vortex possessing an energy $v$, capable, under heat and cold, of adapting itself to changed environment by cumulative indraught and outdraught, i.e. $v^{ \pm_{m}^{n}}$.

In one plane, equal spheres being equitriangularly arranged, each sphere forms a centre capable of supporting, by surface tension, an equal number of spheres around it. In space, the nearest approach to this perfect equilibrium is by means of the five best symmetries, or so-called regular solids, whereof three dominate elemental crystals. ${ }^{3}$ Alike

1 See Barlow and Pope, Chemical Society Transactions, 1907, vol. xci., p. Ir $52 . \quad 2$ Retgers, Zeitsch. phvsical Chem., I894, xivo, I.

NO. 2 I 25 , VOL. 84$]$ as to points, faces, edge-lines, and circum-radial lines, these five contain only the factors 2 and 3 (crystalline) and 5 (non-crystalline), greatly complicated, however, by the last of these :

Crystalline : $\quad$ hex $\sqrt{2: \mathrm{I}} ;$ tet $\sqrt{8: 3} ;$ oct $\sqrt{4: 3}$ Non-crystalline $: i c \sqrt{2\left(1-\frac{\sqrt{5}}{5}\right)}: \mathrm{I} ;$ do $\sqrt{2\left(\mathrm{I}-\frac{\sqrt{5}}{3}\right)}: \mathrm{I}$.

Now the problem of the volutional interconversion (on the principle $v^{ \pm_{m}^{n}}$ ) of the three first ratios 2, 3, and 5, yields to a simple and highly accurate solution, ${ }^{2}$ whereas adding the two last, $i c$ and $d o$, the solution becomes complex; but, on the lines of the simple interconversion, there are contained several approximate interconversions with ic and $d o$, the errors of which are the precise weights of $\mathrm{H}^{1}:{ }^{4}$ by different syntheses :

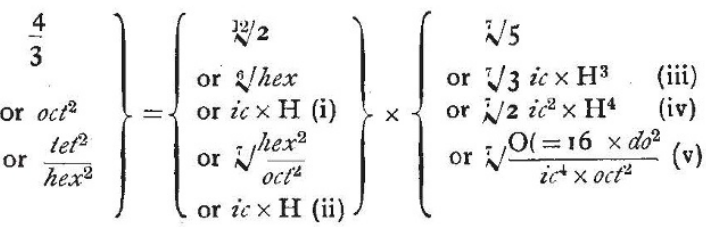

The numbers (i), (ii), (iii), (iv) refer to Morley's four experimental weights of $\mathrm{H}^{3}{ }^{3}$ which the formula hits precisely :

(i) $\mathrm{H}_{2}: \mathrm{O}$ mean $=\mathrm{I} \cdot 0076 \mathrm{r}$

(iii) Gravimetry mean I 00762

(ii) $\mathrm{H}_{2}: \mathrm{O} \max .=\mathrm{I} \cdot 00777$

(iv) $\mathrm{H}_{2}: \mathrm{H}_{2} \mathrm{O}$, , $\mathrm{I} \cdot 00765$

Two basal equations are here involved,

$$
(4 / 3)^{x}=2^{\frac{x}{12}} \times 5^{\frac{x}{7}} \text { and } 2^{x}(5 / 4)^{7 x}=3^{2 x} \times 1 \cdot 5^{\frac{x}{7}} \text { - }
$$

7 and 12 being severally the combinable group and series numbers of the table. The main equation (threes strong) appears accurate to some 50 decimal points; the secondary (fives strong) rather less so. They meet at $\pi / 1 \cdot 5=\sqrt[12]{2}$; with an error of 0.00016 , the crux of the hydrogen ranges. Their great accuracy points to a profound numeric and geometric principle. Hex, hex ${ }^{2} /$ oct $^{2}$, Eoc., compensationvortices cannot evolve to their 6 th and 9 th roots without developing hydrogen, and thereupon compensating ic, \&oc. and, inversely, ic, Sve., cannot involve to their 6th and 7 th powers without ultimately throwing off hydrogen and compensating hex, hex $/$ oct $^{2}$, \& c.

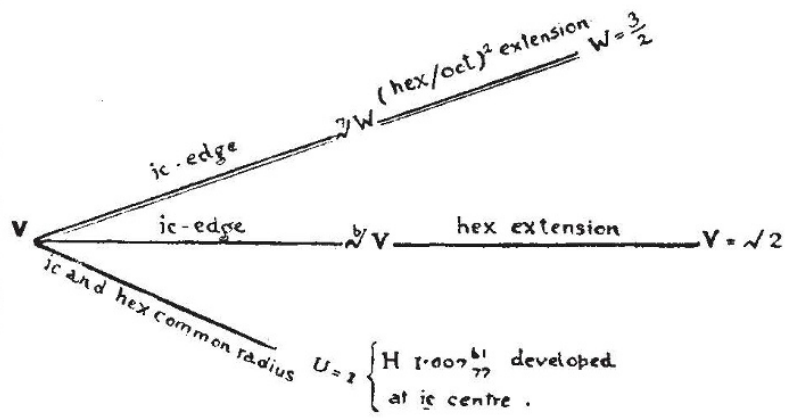

(i) The compensation-vortex at the end of the cubic (or tet/oct) edge-line, pulls, as required, by $\sqrt{ } 2: 1$ against the circum-cube radius. This crystalline symmetry being disturbed by heat, the vortex unravels or evolves to its 6 th root, travelling down the line to the point marked $\sqrt[5]{ } v$. It there precisely compensates the icosahedral edge: circum-

1 Tet, hex, oct, ic, and do here stand for the ration, or the weights compenting the ratios, edge-line: circum-radius (i.e. the radius of a circumpensating the ratios, edge-line:circum-radius

2 A log-algebraic problem of eight terms unknown, it was soluble only by 2 A log-algebraic problem of eight terms unknown, it was soluble only by
reference to vhilosophical considerations anterior to those now discussed.

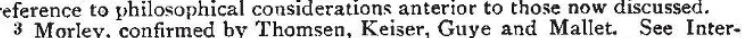
3 Morley, confirmed by Thomsen, Keiser, Guye and Mallet. See Inter-
national Committee's Report, Chemical News, February 12,1897 , May 5 , national Committee's Report, Chemical News, February ז2, 1897 , May 5,
1899, June r r, 1897, and May 12, 1905; or Freund's "Chemical Composition," 1904, p. 220 . 